\title{
Bevirimat Dimeglumine
}

National Cancer Institute

\section{Source}

National Cancer Institute. Bevirimat Dimeglumine. NCI Thesaurus. Code C78045.

A dimeg lumine formulation of bevirimat, a drug derived from a betulinic acid-like compound, first isolated from the Chinese herb Syzygium claviflorum, with activity against human immunodeficiency virus (HIV). Bevirimat acts by binding to the Gag capsid precursor protein and blocking its conversion to mature capsid protein by protease cleavage. It potently inhibits replication in both wild-type and drug-resistant (reverse transciptase or protease) HIV-1 isolates. 